

Revista de Derecho

ISSN: 2313-6944

ISSN: 2707-9651

revistaderecho@unap.edu.pe

Universidad Nacional del Altiplano

Perú

\title{
PROBLEMAS EN LA IMPLEMENTACIÓN DE LA LEY DE SALUD MENTAL
}

Ñaca Ramírez, Davis Rurik Van

PROBLEMAS EN LA IMPLEMENTACIÓN DE LA LEY DE SALUD MENTAL

Revista de Derecho, vol. 5, núm. 2, 2020

Universidad Nacional del Altiplano, Perú

Disponible en: https://www.redalyc.org/articulo.oa?id=671870937007

DOI: https://doi.org/10.47712/rd.2020.v5i2.62

\section{(c) (1)}

Esta obra está bajo una Licencia Creative Commons Atribución 4.0 Internacional. 


\title{
PROBLEMAS EN LA IMPLEMENTACIÓN DE LA LEY DE SALUD MENTAL
}

Problems in the implementation of the mental health law

Davis Rurik Van Naca Ramirez

Universidad Nacional del Altiplano de Puno, Perú

\author{
DOI: https://doi.org/10.47712/rd.2020.v5i2.62 \\ Redalyc: https://www.redalyc.org/articulo.oa? \\ id $=671870937007$ \\ Recepción: 07 Julio 2020 \\ Aprobación: 25 Octubre 2020 \\ Publicación: 27 Octubre 2020
}

\section{RESUMEN:}

En la actualidad existe la necesidad de identificar los problemas que existe en la sociedad entorno a su salud mental y estudiar los problemas de la implementación de la Ley de salud mental $\mathrm{N}^{\circ} 30947$ en el Perú, esto porque será un estudio de la realidad peruana el único camino que nos permita plantear mejoras a la Ley y formular políticas públicas e institucionales en los diferentes niveles de gobierno. En ese contexto es importante traer a mención que nuestro país es uno de los que está dentro del grupo de Estados que tienen ciudadanos que requieren atención de su salud mental de manera preventiva y asistencial. Pero también ocurre que curiosamente que somos uno de los países con menos infraestructura adaptada para estas necesidades en los hospitales, por eso es allí donde radica la importancia de profundizar los problemas latentes, sin dejar de lado que el equipo que trabajará dentro de este debe tener una adecuada capacitación para su trabajo y tener pleno conocimiento de los derechos que están en la esfera de los pacientes. Es así que este estudio tiene por objetivo abordar estos problemas vinculados a la implementación de salud mental en nuestro país.

Palabras Clave: Salud mental, Problemas, Realidad Nacional.

\section{Abstract:}

Currently there is a need to identify the problems that exist in society around their mental health and study the problems of the implementation of the Mental Health Law No. 30947 in Peru, because this will be a study of the Peruvian reality on The only way that allows us to propose improvements to the Law and formulate public and institutional policies at different levels of government. In this context, it is important to bring up a mention that our country is one of the group of States that have citizens who require preventive and welfare mental health care. But it also happens that curiously we are one of the countries with the least adapted infrastructure for these needs in hospitals, that's why there is the importance of deepening latent problems, without neglecting the team that works within these obligations to have adequate training for their work and having full knowledge of the rights that are in the sphere of patients. Thus, this study aims to address these problems related to the implementation of mental health in our country.

KEYwORDs: Mental Health, Problems, National Reality.

\section{INTRODUCCIÓN}

En el presente trabajo se abordara la importancia de conocer la salud mental para su implementación en el Estado peruano, es así que su estudio implica hacer referencia al bienestar, autonomía, y reconocimiento de la habilidad de realizarse emocionalmente e intelectualmente, también estudiar la reciente Ley de Salud Mental, estudiar los principios de la salud mental que deben guiar las acciones del Estado en su implementación y en ese razonamiento observar cuales son los problemas más importantes que afronta la implementación de la referida Ley $\mathrm{N}^{\circ} 30947$. En ese sentido siendo conocedores de la realidad no debemos olvidarnos de que vivimos en un país donde existen grupos humanos de diferentes clases sociales que están en un estado de necesidad y que requieren ser atendidos en todo lo concerniente a salud mental .Pero sobre este punto tampoco deben pasar desapercibidos que dentro de estos grupos, también hay personas que padecen problemas de salud mental y que requieren de instalaciones especiales para su cuidados, en ese contexto el creciente conocimiento sobre esta interdependencia mencionada, surge de manera más reciente y los estudios 
recientes de especialistas en la materia nos llevan a concluir que el problema de salud mental es un pilar central en el bienestar general de los individuos, sociedades y naciones. Por tanto su estudio resulta relevante y se desarrolla a continuación.

\section{DESARROLLO}

\subsection{SALUD MENTAL}

Para iniciar el tema es pertinente conocer que se debe entender por esos términos y en ese aspecto el Departamento de Salud Mental y Abuso de Sustancias (2004) indica "que la Salud mental es más que la mera ausencia de trastornos mentales” (pág. 7). Por otro lado también hace mención de la dimensión positiva de la salud mental, la cual ha sido subrayada en la definición de salud de la OMS, tal cual consta en la constitución mismas de varios Estados se concibe a la salud como un estado de completo bienestar físico, mental y social y no solamente la ausencia de afecciones o enfermedades.

Mientras que la Organización Mundial de la Salud (2004), describe a la Salud Mental como "un estado de bienestar en el cual el ciudadano percibe sus propias aptitudes, puede afrontar las presiones normales de la vida, puede trabajar productiva y es capaz de hacer una contribución a su comunidad".(pág. 11). En ese sentido la salud mental se concibe como la base para el bienestar y el funcionamiento efectivo de un individuo y una comunidad. (Recuperado de http://acp.org.pe/salud/salud-mental-estado-de-bienestar/). En ese contexto la Organización Mundial de la Salud (2004) apoyando las ideas expuestas también refiere que "mucho más que la ausencia de una enfermedad mental, ya que las condiciones y capacidades mencionadas en la definición, tienen valor por sí mismas. Ni la salud física ni la salud mental pueden existir solas, además el funcionamiento de las áreas mentales, físicas y sociales son interdependientes" (pág.12)

La Organización Panamericana de la Salud (2001) sostiene que la salud mental es" el núcleo de un desarrollo equilibrado de toda la vida, que desempeña una función importante en las relaciones interpersonales, la vida familiar y la integración social. Si bien es el núcleo de desarrollo equilibrado y es importante para las relaciones interpersonales, el Estado debería preocuparse para que los ciudadanos acudan a centro hospitalarios cada vez que se sientan mal y así lograr que en futuro puedan hacer la integración social a la que se refiere, en ese orden de ideas tenemos que también se la considera a esta como un factor clave para la inclusión social y plena participación en la comunidad y en la economía. Gonzales (2016) hace bien "en afirmar que en realidad, la salud mental es mucho más que la mera ausencia de enfermedades mentales, es una parte indivisible de la salud y la base de bienestar y el funcionamiento eficaz de las personas."(pág.62)

También ha sido definido como un estado de bienestar por medio del cual los individuos son capaces de hacer frente al estrés normal de la vida, trabajar de forma productiva y fructífera, y contribuir a sus comunidades. (El Departamento de Salud Mental y Abuso de Sustancias, 2004). Por otro lado la Salud mental se refiere a la posibilidad de acrecentar la competencia de los individuos y comunidades y permitirles alcanzar sus propios objetivos. Salud mental hasta aquí descrita es materia de interés para todos, y no sólo como algunos creen, que es para aquellos afectados por un trastorno mental. Por ello también se considera que en todos los individuos, la salud mental, la física y la social están íntimamente enlazadas y es el Estado al ver la realidad el que debería preocuparse por plantear soluciones rápidas y modernas. Pero desafortunadamente, en la mayor parte del mundo, no se le otorga a la salud mental y a los trastornos mentales la misma importancia que a la física y por esos hechos han sido más bien objeto de ignorancia o desatención.

\subsection{PRINCIPIOS DE LA LEY DE SALUD MENTAL}

Los principios tratados en las Naciones Unidas sobre la protección de los enfermos mentales, así como el mejoramiento de la atención de Salud mental establecieron estándares mínimos de derechos que deben practicarse en el campo de la salud mental y de los derechos humanos (Gonzales, 2016). Refirieron también que los órganos internacionales de monitoreo internacional han empleado los Principios EM como interpretación autorizada de las exigencias de convenciones internacionales tales como el PIDESC. Gonzales 
(2016) refiere que estos principios han servido también como una guía para el desarrollo de la legislación de salud mental en muchos Estados.

Estos estados han incorporado en sus sistemas jurídicos las propias leyes sobre salud mental. En ese sentido los Principios que establecen estándares sobre el tratamiento y las condiciones de vida en instituciones de salud mental crean protecciones contra la detención arbitraria en esas instituciones. La utilidad de estos principios es que se aplican en general a las personas con trastornos mentales, estén o no internadas en instituciones del tipo psiquiátricas, y a toda persona admitida en una institución psiquiátrica que en momento ha sido o no diagnosticada como portadora de un trastorno mental.

Esta última disposición es importante porque muchas partes del mundo se usan las instituciones de salud mental de larga duración en su aplicación, es decir tienen como depósitos de personas que no tienen una historia de trastorno mental, o que actualmente no tienen trastornos mentales, pero que permanecen en la institución debida a la falta de otras instituciones o servicios comunitarios que les permitan satisfacer sus necesidades. Los Principios reconocen que toda persona con trastornos mentales tiene derecho a vivir y a trabajar, en la mayor medida posible, en la comunidad.

Algo importante sobre los Principios que refiere el Secretario General de la ONU es que estos "ofrecen en algunos casos un grado menor de protección que el ofrecido por los tratados de derechos humanos existentes, por ejemplo, en relación a exigencia de consentimiento informado previo al tratamiento". (Naciones Unidas, 2003).

En este sentido, algunas organizaciones han cuestionado la protección proporcionada por los Principios (y, en particular, por los principios 11 y 16) y su concepción con los estándares de derechos humanos existentes para el tratamiento y la detención involuntaria."(Naciones Unidas, 2003)

Para tener un panorama despejado de lo que referimos es preciso citar los principios y enfoques transversales más importantes que desarrolla, en su artículo 13, la Ley N 30947 "Ley de Salud Mental"

1. Accesibilidad. Se busca el acceso a todas las personas, sin discriminación, a las acciones de promoción, prevención, atención y rehabilitación en salud mental.

2. Calidad. El Estado garantiza en la medida de sus posibilidades garantiza el acceso a los servicios brindados en los establecimientos de salud, de acuerdo a su capacidad de resolución y niveles de atención

3. Derechos humanos. Las implementaciones de estrategias, deben adecuarse a la Convención sobre los Derechos de las Personas con Discapacidad y a otros instrumentos internacionales y regionales de derechos humanos que operan en el Perú

4. Inclusión social. La atención, cuidado y tratamiento de un problema de salud mental busca la inclusión de la persona en su familia y en la sociedad a la que pertenece.

5. Interculturalidad. El Estado promueve un trato entre los diferentes grupos culturales de manera cordial y a la par.

6. Enfoque de discapacidad. El Estado busca adoptar medidas, para eliminar las puertas que impidan el ejercicio completo de la salud mental de las personas con discapacidad.

\subsection{CAMBIO DE ENFOQUE DE LA SALUD MENTAL}

Históricamente podemos remontarnos a los primeros indicios de cambio en la concepción y estructuración de la reglamentación en salud mental en Europa porque allí en Italia surgió la Ley 180 que su momento extendió la tutela de la salud mental a todos los Italianos sin distinción de clase social o económica y al hacer esto, busco cambiar la idea de que los manicomios son la única solución, lo cual implicó el paso de una terapia de psiquiatría de la cura a una psiquiatría del cuidado de la salud mental poblacional, reemplazando el control social por un abordaje terapéutico (Gonzales,2016)

Es en ese sentido que el rol de la familia tiene una función importante y asi lo afirma el Departamento de Salud Mental y Abuso de Sustancias (2004) que refiere que "los miembros de la familia son, con frecuencia, los cuidadores primarios de las personas con trastornos mentales. Como tal, ellos proveen apoyo emocional e instrumental, y con no menor frecuencia también deben afrontar los costos financieros asociados con el 
tratamiento y la atención. Se estima que una de cada cuatro familias tiene por lo menos un miembro afectado por un trastorno mental o conductual".

\subsection{DISCRIMINACION Y NECESIDADES DE LOS PACIENTES PSIQUIATRICOS}

Gonzales (2016) refiere que "los pacientes psiquiátricos necesitan recibir atención médica de calidad protegidos de todo tipo de discriminación”. Es así que si bien la Ley de Salud Mental propone que su proceso de atención se realice preferentemente a los más afectados, no debe olvidarse que en el marco de un abordaje interdisciplinario e intersectorial este también debe ser organizado y es lo que más hace falta en nuestro país" (pág.60). Esto se plantea a fin de que basado en los principios de la atención primaria de la salud se logren. Si bien la ley propone la creación de instituciones complementarias, ocurre que en la práctica su disponibilidad es limitada en relación a la demanda total de un país como el nuestro, por lo cual los pacientes que no cuentan con un núcleo familiar y afectivo pueden terminar en situación de desamparo, es así que esta posibilidad no está contemplada en la ley y debe regularse.

El Departamento de Salud Mental y Abuso de Sustancias (2004) también indica que "las personas con trastornos mentales con frecuencia sufren un amplio espectro de violaciones de sus derechos humanos y de estigma social" (pág.12).

Pero bien ahora veamos la realidad nuestro país, en un reciente estudio de experiencias en la implantación de la reforma de atención en Salud Mental en América latina e Iberoamérica se sostuvo que "en el Perú la atención de la salud mental ha sido olvidada, habiéndose priorizado el socorro de la salud física bajo el supuesto de que los problemas vinculados a esta realidad generan mayor mortalidad. Sin embargo, en nuestro país año a año se pierden la vida muchas personas por suicidio y el $20 \%$ de la población adulta y adulta mayor padece de un trastorno mental, especialmente depresión, trastorno de ansiedad y alcoholismo" (pág. 24). Esto aun sin considerar a quienes sufren de alguna violencia; también refiere que además del $20 \%$ de niños y niñas que padecen de algún trastorno mental como los problemas severos de comportamiento y problemas de aprendizaje. El 90\% de esta población no recibe atención, siendo mayor esta cifra en población pobre y extremadamente pobre. (Ministerio de Salud, 2017)

Detallado esto, se observa del estudio que los problemas involucrados a la salud mental también tienen unos puntos de contactos con el aprendizaje, depresión, problemas de comportamiento y que el Estado debería construir centros hospitalarios donde se proporcione atención antes que empeoren los problemas y sean irreparables en los ciudadanos.

Esta información es corroborada por Ocaña (2015) el cual refiere que en el Perú, se estima que más de un tercio (37\%) de la población sufre o ha sufrido un trastorno de salud mental, pero son las mujeres quienes padecen más estos trastornos en comparación con los hombres, además de ser ellas quienes en su mayoría tienen la responsabilidad de los cuidados a las personas que padecen un trastorno de salud mental crónico.

Además, debemos conforme refiere el Ministerio de Salud (2004) que "los trastornos mentales comprenden cinco de las diez causas principales de la carga de morbilidad en todo el mundo, es así que, la salud mental se ha planteado como una problemática de Salud Pública que exige una visión interdisciplinaria y multisectorial por parte del Estado". En ese sentido existe la necesidad de tutela de derechos en la esfera de los derechos humanos que podrían ser menoscabados en personas con problemas de salud mental en nuestro país.

\subsection{PROBLEMA DE PACIENTES QUE SE QUEDAN EN INSTALACIONES DE HOSPITALES POR SER UN PELIGRO.}

Es conocido que existen en la ejecución de ilícitos penales algunos que sujetos activos que están dentro de este grupo que requiere atención por la salud mental que tienen y por tanto deben tener un trato diferente, esto es apoyado por Gonzales (2016) que refiere "los pacientes psiquiátricos judicializados que, dada la inexistencia de instituciones destinadas a su asilo, deben permanecer en los hospitales generales bajo normas estrictas de seguridad, adicionando una responsabilidad al rol de los profesionales de la salud y sanatorios" (pág.60). Si bien se espera que la reforma mejore la calidad de vida de los pacientes, si la 
articulación entre los agentes del sistema de salud (hospitales, centros de día y profesionales de la salud) es deficiente, sus logros pueden no ser los deseados.

Muchos establecimientos para tratamientos psiquiátricos, hospitales generales e instituciones sociales en ciertos países hasta la actualidad siguen usando camas tipo jaulas para restringir el movimiento de personas con trastornos mentales y problemas mentales. Las camas tipo jaulas pueden tener una red de fuerza o, en algunos casos inclusive, barras metálicas que sirven para inmovilizar al paciente (Departamento de Salud Mental y Abuso de Sustancias, 2004). Asimismo ocurre que en oportunidades, los pacientes permanecen en estas camas tipo jaulas por periodos prolongados, hasta años y que esto debe cambiar si se piensa implementar de manera adecuada la Ley de Salud Mental. Este tipo de instrumentos son frecuentemente usados cuando el personal es escaso o está mal capacitado, en otras oportunidades se les usa como castigo o como amenaza de castigo.

Ocoña (2015) refiere que "algunas situaciones en la relación médico a cuidadora y esta al paciente han sido percibidas como maltrato por parte de los médicos u otros profesionales. El maltrato al parecer se percibe más cuando es hacia la cuidadora, o cuando este es muy evidente colindando con la negligencia en el tratamiento" (pág.132). Detallado esto se tiene indicios que también es un problema latente en los centros de atención que requiere una regulación.

\subsection{PROBLEMA DE COMPARTIR EL AMBIENTE EN LEY DE SALUD MENTAL.}

La Ley de salud mental involucra varias políticas que requieren implementarse para su logro, una de ellas es que los ambientes destinados únicamente a atención de todo tipo de enfermos tengan en el futuro un lugar para atender a estos, pero por otro lado también deben construirse nueva infraestructura, hasta allí no hay problema. Pero en donde si habrá es en el presupuesto destinado para estas edificaciones, es así que, algunos autores como Gonzales (2016) refieren que "en la realidad en la que se encuentra nuestro país ocurre que podría darse que a "partir de la implementación de la Ley de Salud Mental otros pacientes que demanden atención de la salud con patologías diferentes a los trastornos mentales y deben compartir espacios del sector salud, como hospitales generales o centros de atención primaria de la salud, con personas que sufren trastornos psiquiátricos". (pág. 61) Asimismo otro fenómeno es que estos pacientes podrían sentir temor o aversión a compartir las instalaciones, lo cual debe tenerse en cuenta como parte de las medidas de adaptación.

La necesidad de implementar medidas y programas es urgente en nuestra realidad esto es apoyado por los Estudios Epidemiológicos en Salud Mental que desarrolla el Instituto Nacional de Salud Mental Honorio Delgado Hideyo Noguchi (2013) el cual refiere "que la presencia de al menos una discapacidad o inhabilidad en la población peruana es en promedio del 14\% desde el año 2002 oscilando entre 8,3\% en las Sierra Rural del Perú hasta 26.5\% en Lima y Callao" .(pág. 17)

Por otro lado, también dicho estudio refiere que en lo referente a como son tratadas estos pacientes con trastorno mental por la población, se evidenció que la aceptación hacia ellas oscila desde un $13.3 \%$ en ciudades de la sierra rural de nuestro país hasta $35,1 \%$ en ciudades de la costa peruana; el comportamiento de dar ayuda hacia estas personas con trastorno estuvo presente desde en más del $40 \%$ de ciudadanos de la costa peruana y no sobrepaso el $60 \%$ en ciudades de la sierra rural

\subsection{RESPONSABILIDAD DE LOS PACIENTES}

Con respecto a los profesionales de la salud las normas legales de otros lugares indican que la atención a los pacientes debe ser hecha por un equipo interdisciplinario de salud mental, integrado por un médico (para el cuidado de la salud), un psicólogo (para evaluar la dimensión subjetiva), un trabajador social (para considerar las condiciones sociales) y un enfermero (para los cuidados cotidianos). En ese sentido Gonzales (2016) indica que en ese "contexto para un adecuado tratamiento se debe garantizar la existencia del equipo y si bien el médico psiquiatra continúa teniendo la responsabilidad civil y penal por cada paciente, en los hechos su autoridad se ve restringida dado que cualquier decisión de tratamiento o internación debe ser acordada con el resto del equipo de atención, lo que produce una inconsistencia de esta legislación con las leyes del ejercicio profesional". 


\subsection{HOSPITALES EN PERÚ SU IMPORTANCIA EN SALUD MENTAL}

Los hospitales generales se conciben como instituciones públicas y/o privadas oferentes de servicios de salud que deben realizar redistribuciones de espacio y lugares para los pacientes y usuarios, así también deben distribuir los lugares de servicios y recursos humanos para responder a las necesidades de atención de los pacientes con enfermedades mentales (Gonzales, 2016). Esto implica, una reducción de los recursos en otras áreas del hospital, a cambio de destinar más fondos a la creación de espacios que garanticen la tranquilidad y el desarrollo de las actividades terapéuticas de los pacientes psiquiátricos tales como clases de música, teatro, charlas grupales, danza, entre otras. Si no disponen de partidas presupuestarias adicionales, esta movilización de recursos puede llegar a perjudicar la oferta de servicios para los pacientes que sufren otras patologías distintas a las psicopatías.

En ese contexto el Departamento de Salud Mental y Abuso de Sustancias (2004) refiere que "una combinación adecuada de programas de tratamiento y prevención en el campo de la salud mental, en los marcos de estrategias públicas generales, puede evitar años vividos con discapacidad e, incluso, la muerte prematura, reducir el estigma que rodea a las enfermedades mentales, aumentar considerablemente el capital social, ayudar a reducir la pobreza y a promover el desarrollo del país".

El presidente de la Asociación de Psiquiatras de América Latina, planteó que la experiencia internacional más sólida en el área aconseja la transformación gradual de los hospitales monovalentes, para dotarlos de recursos que sean modernos y adecuados y así garantizar la mejor atención posible. Uno de los peligros, no infrecuente, es que se clausuren los servicios hospitalarios monovalentes sin alternativas sustentables en la red asistencial. Vinculó esto al hecho de que actualmente los hospitales generales no cuentan con las camas de internación necesarias

Hermosilla (2012) indica sobre la importancia de los hospitales que "muchos sostuvieron que los hospitales monovalentes deberían continuar existiendo, adaptándolos. Otras voces, sumaron a los obstáculos presupuestarios, razones culturales, ideológicas, que generarían que no estén dadas las condiciones para el cierre de los hospitales monovalentes" (pág. 138). En ese contexto se debería diferenciar y definir qué no es, o al menos no debería ser desinstitucionalizar: no es abandonar a los pacientes, dejarlos en la calle, dejarlos sin atención. Hermosilla (2012) sobre esto también refiere que "tampoco es negar la internación, en los casos en los que realmente es necesario, como lo plantearon sus colegas al decir que la Ley no se está refiriendo a expulsar a los pacientes, sino a refuncionalizar el sistema”.(pág.138).En este sentido, es importante que la norma consiga su reglamentación, ya que se podría esclarecer este punto, garantizando que la desmanicomialización no se realice con perjuicio de las personas institucionalizadas.

La Ley de Salud Mental en concordancia con muchos Psicólogos propone la creación de centros intermedios y la coordinación con centros de atención primaria de la salud. Dado que las patologías psiquiátricas generan síntomas que aíslan al paciente de su familia y comunidad, en muchos casos éstos carecen de toda conciencia de su enfermedad y situación, motivo por el cual suelen abandonar los tratamientos. Estos hechos tornan necesaria la creación de espacios terapéuticos intermedios, con abordaje diario y grupal, que promuevan su compensación, rehabilitación y estabilización, así como el aprendizaje de conductas y hábitos sobre el manejo de su enfermedad. Estos centros se deberán crear y/o adaptar progresivamente en las diferentes regiones del país.

Finalmente es necesario mencionar que en Perú al 2008 existen 21 hospitales generales (unidades con servicios de psiquiatría de base comunitaria) que ofrecen servicios en psiquiatría. De este total, 10 pertenecen al MINSA y 6 a EsSalud. Entre aquellos pertenecientes al MINSA que tenían pacientes hospitalizados en el 2007 tenemos:

- Hospital Aplao, Arequipa

- Hospital Honorio Delgado, Arequipa

- Hospital Departamental Ica, Ica

- Hospital de Apoyo Domingo Olavegoya, Junín 
- Hospital de Apoyo Manuel Higa Arakaki

- Hospital Regional Docente de Trujillo, La Libertad

- Hospital Regional de Puno (Manuel Núñez Butrón), Puno

- Hospital Hipólito Unanue, Tacna

Señala Informe de Salud Mental del Sub Sector del Ministerio de Salud en el Perú (2008) ocurre que "pese a que existe la norma de atención de salud mental según niveles de complejidad (Resolución Ministerial No 0074-84-SA/DVM), la que estipula que el número de camas para hospitalización psiquiátrica no debe ser menor del $5 \%$ del total de camas del hospital regional, general y local", la reliada es diferente y además menciona que "tasa de ocupación es de 4 camas por cada 100.000 habitantes y que en ell 2007, la cantidad de pacientes atendidos en las instituciones psiquiátricas ha sido de 19.886. Un dato importante también es mencionar que en las instituciones psiquiátricas, $8 \%$ de los pacientes estuvieron internados por lo menos un año; $2 \%$, entre uno y 4 años; 3\%, entre 5 y 10 años; y mas del 40\%, un tiempo superior al de 10 años, según el informe estadístico específico solicitado a cada hospital en el 2007”. Siendo eso así cabe resaltar la existencia de un problema que afecta el cuidado de la salud mental.

\subsection{RECONOCIMIENTO DEL DERECHO A LA SALUD COMO DERECHO AUTÓNOMO}

Leon (2014) "Uno de los primeros temas a los que se enfrentó el Tribunal al delinear los alcances del derecho a la salud fue el relacionado con su carácter de derecho fundamental" (pág.393).Asimismo indica el autor que "cuando la Constitución de 1993 desde un inicio prescribe que todos tienen derecho a la protección de su salud, el Tribunal fue receptor de alguna jurisprudencia comparada que solo reconoce el carácter ius-fundamental del derecho a la salud cuando este se encuentra engarzado con el derecho a la vida o a la integridad física”. (León, 2014, pág. 393) pero por otro lado la Sentencia N 5842-2006-PH C/TC indica que "esta postura queda registrada tempranamente, por ejemplo, en el caso Juan Islas Trinidad y otros, o en el caso del Penal de Challapalca, donde el Tribunal ubica este derecho en la cláusula de los derechos innominados merced a su vinculación con el derecho a la vida, a la integridad y a la dignidad de la persona. Posteriormente ratifica este criterio en el caso Azanca Alhelí Meza García, donde a pesar de que se desarrolla este derecho en el marco más amplio de la dogmática de los derechos sociales, su carácter ius-fundamental solo lo reconoce cuando están en juego otros derechos fundamentales, básicamente la vida. Sin embargo, en el caso José Luis Correa Condori, el Tribunal reconoce tímidamente que el derecho a la salud tiene una categoría ius-fundamental autónoma, aunque persiste en el criterio de que dicho fundamento depende en una importante medida de su relación con el derecho a la vida”.(Sentencia TC 5842,2006)

Por otro lado también en el Informe de Salud Mental del Sub Sector del Ministerio de Salud en el Perú (2008) se indica que "se estima que entre $2 \%$ y $6 \%$ de los pacientes internados en las instituciones psiquiátricas fueron restringidos es decir, se les colocó algún dispositivo físico que no podían quitar con facilidad o aislados colocados en solitario en un espacio confinado por lo menos una vez en el último año. Se desconoce esta cifra en el caso de los hospitales generales (unidades de hospitalización psiquiátrica de base comunitaria)(pág. 17).

Pero tal vez lo que resalta de este informe y tiene trascendencia para la investigación emprendida es que menciona "la dificultad de acceso a los servicios de salud mental para los usuarios pertenecientes a minorías lingüísticas, étnicas y religiosas también es un aspecto que debe ser estudiado por las autoridades de salud correspondientes, más aún cuando no se conoce un planteamiento sobre cómo abordar esta situación tomando en cuenta las creencias y el lenguaje de estos pacientes, además de que no se cuenta con estadísticas nacionales al respecto."(pág. 17). En esa línea se observa que existe el conocimiento de la afectación del acceso a servicios básicos de salud en el cuidado de la salud mental.

\subsection{PROBLEMAS POSTERIORES A LA PROMULGACIÓN DE LA LEY DE SALUD MENTAL}

En el Perú, uno de los principales problemas para acceder a una atención de salud mental es el aspecto económico. Es por ello que esta ley establece que las personas en condición de pobreza o pobreza extrema que sufran algún tipo de afección mental y no cuenten con seguros serán incluidas en el Seguro Integral de 
Salud (SIS). Además, los seguros de salud públicos y privados deberán cubrir este tipo de atención dentro de sus planes, incluyendo tratamiento ambulatorio u hospitalización, así como acceso a medicamentos. Las compañías privadas de seguros estarán obligadas a brindar cobertura para el diagnóstico, tratamiento y rehabilitación de los pacientes.

Sin embargo, esta ley presenta un problema, cuando se indica que los profesionales quienes deberían atender a los pacientes en salud mental, son los psiquiatras y psicólogos, pero la ley ha excluido a estos últimos. Es así que, en su Artículo 25 sobre esta materia refiere que la competencia para el diagnóstico, establece: "El diagnóstico y la determinación de la existencia de un problema de salud mental debe realizarse por un médico psiquiatra colegiado con apoyo técnico profesional del equipo de salud mental; y, en ausencia de complemento, por médico cirujano colegiado, de acuerdo a las normas técnicas aceptadas internacionalmente" (Ley 30947,2019). En este sentido, teniendo en cuenta lo establecido en la Ley del Trabajo del Psicólogo Ley $\mathrm{N}^{\circ}$ 28369, se tiene que señala: "el psicólogo es aquel profesional que estudia y trata la conducta humana con competencias en la prevención, promoción, diagnóstico, tratamiento y recuperación de la salud mental de la persona humana, la familia, la sociedad en el ámbito psicosocial"; de lo señalado podemos precisar que debió considerárseles también a los profesionales de psicología para poder dar un diagnóstico de trastornos mentales, siempre dentro de los límites de su competencia profesional, más en lo referente a la medicación el más apropiado es el psiquiatra.

\section{CONCLUSIONES}

Se observa la necesidad de crear un Órgano de Revisión que supervise los servicios de salud mental y vele por la protección de los derechos humanos de aquellos usuarios con una salud mental vulnerable. A su vez, resulta imperioso desarrollar planes de prevención en materia de salud mental y programas específicos de inserción de los ciudadanos en la sociedad y en ámbito laboral, esto para personas con padecimiento de posibles problemas mentales futuros.

Por otro lado debe reforzar a la célula básica de la sociedad es decir a la familia del paciente psiquiátrico en razón a que es un actor que deberá adaptarse a este nuevo proceso ya que la externación un familiar psiquiátrico le demandará tiempo para atenderlos, traslados y de cuidados. Si bien la iniciativa en la Ley de Salud Mental propone entra varias cosas no sería menos importante complementar la contención familiar con servicios de atención ambulatoria, esto sin duda genera un reacomodamiento de la dinámica del hogar, mayores gastos de bolsillo, y una gran incertidumbre pues los dispositivos intermedios son escasos y aún no funcionan óptimamente en todo el país, produciendo una demanda insatisfecha de tratamientos.

Finalmente es necesario construir nuevos hospitales a nivel de todo el Perú a fin de que los ciudadanos concurran a estos ambientes a recibir un tratamiento preventivo o asistencial. En ese sentido necesitamos que se derive un mayor presupuesto para este tipo de edificaciones y de esa forma no se altere los ambientes destinados a cuidados ya predeterminados, es decir no se acomode de manera apresurada la los ambientes que ya tiene un uso. También debe regularse de mejor forma las actividades encomendadas a cada profesional y a los equipos de trabajo que se ocuparan de hacer cumplir la ley sobre salud mental.

\section{BIBLIOGRAFÍA}

Hermosilla (2012), Ley De Salud Mental 26657. Antecedentes Y Perspectivas, Revista Latinoamericana de Ciencia Psicológica, Buenos Aires.

Instituto Nacional de Salud Mental "Honorio Delgado - Hideyo Noguchi (2013), Estudio Epidemiológico de Salud Mental de Lima y Callao - Replicacion, Editorial Instituto Nacional de Salud Mental,Lima.

Ministerio de Salud (2008),Informe de Salud Mental del Sub Sector del Ministerio de Salud en el Perú, recuperado el 30 de junio del 2020 de http://bvs.minsa.gob.pe/local/MINSA/1246_MINSA1502.pdf 
Leon, F. (2014). El derecho a la salud en la jurisprudencia del Tribunal Constitucional peruano. Pensamiento Constitucional, Editorial de PUCP,Lima

Organización Mundial de la Salud (2004), Promoción de la Salud Mental, Editorial Departamento de Salud Mental y Abuso de Sustancias de la Organización Mundial de la Salud, Ginebra.

Ocaña Clarisa (2015). Salud Mental para Todos, Pero Asunto de Ellas. La Salud Mental Desde la Perspectiva de Mujeres-Cuidadoras, Editorial Repositorio de la Universidad Nacional Mayor de San Marcos, Lima.

Gonzales Gisela (2016), Revista de la Asociación Médica de Bahía Blanca,Editorial Instituto de Investigaciones Económicas y Sociales del Sur,Buenos Aires.

Ministerio de Salud.(2017) Experiencias en la implementación de la reforma de la atención en Salud Mental en América Latina e Iberoamérica: Retos y Alternativas". Editorial Ministerio de Salud -Dirección de salud Mental, Perú.

Ovidio C \& Restrepo D \& Cardona D (2016) “Construcción del concepto de Salud Mental Positiva”, Editorial Revista Panamericana de Salud Publica

Salud Mental (2019), Estado de bienestar, Recuperado de http://acp.org.pe/salud/salud-mental-estado-de-bienestar/ en fecha 10 de diciembre del 2019

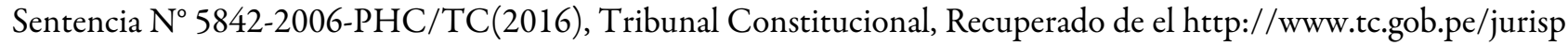
rudencia/2009/05842-2006-HC.pdf en fecha 13 de diciembre. 